\title{
Eine automatische Apparatur zur örtlich und zeitlich aufgelösten Spektroskopie an Funkenentladungen
}

\author{
H. Tholl, I. Sander und H. Martinen \\ Institut für Angewandte Physik der Universität Hamburg \\ (Z. Naturforsch. 25 a, 412-420 [1970] ; eingegangen am 17. Dezember 1969)
}

\begin{abstract}
An automatical recording spectroscopic arrangement was developed to measure the side on distributions of the emitted light of spark channels at different times of the channel development. The radiation at two wavelengths was detected by means of two photomultipliers. The photomultiplier currents are applied to a two channel sampling unit which measures the amplitude of this currents at a fixed selected point of time. The output voltages of the two sampling channels are applied to the $x$-inputs of two $x y$-plotters. By means of an image converter and a television scanning system it was possible to determine that part of the spark channel from which the photomultiplier had received the light. The scanning system produces an output voltage which is proportional to the radial distance of this observed part from the channel axis. This voltage is given to the $y$-inputs of the two $x y$-plotters. The spark channels are initiated "shot by shot" using a pulse generator, and the $x y$-plotters write down the side on distribution "dot by dot" for a fixed point of time. A programmable control and regulating system checks the whole measuring process.
\end{abstract}

\section{Einleitung}

In früheren Arbeiten ${ }^{1,2}$ wurde damit begonnen, Elektronendichte- und Temperaturverteilungen in Funkenkanälen zu bestimmen. Zur Ermittlung dieser Größen war es notwendig, durch spektroskopische side-on-Messungen die Intensitätsverteilung des Funkenlichtes senkrecht zur Funkenachse bei verschiedenen Wellenlängen und zu verschiedenen Zeitpunkten der Funkenentwicklung zu ermitteln. Bei solchen Messungen tritt eine große $\mathrm{Zahl}$ von veränderbaren Parametern auf. Bei der Untersuchung von Funkenkanälen unter fest vorgegebenen experimentellen Bedingungen (z. B. Gas, Druck, Spannung, Entladungsenergie) treten in den Radialverteilungen als freie Parameter noch die Wellenlänge $\lambda$ und die Zeit $t$ auf. In Funkenkanälen tritt in der Regel jedoch auch noch eine ausgeprägte Achsenstruktur auf, so daß als weiterer Parameter der jeweils beobachtete Achsenabschnitt hinzu kommt. Ist es nun erwünscht, die experimentellen Bedingungen in weiten Bereichen zu verändern, so ergibt sich schließlich, daß bei manueller Durchführung, wie z. B. in ${ }^{2}$, infolge der Vielzahl der zu messenden Verteilungen sowohl die Meßzeit als auch die Fehlermöglichkeiten zu groß werden. Da die Bestimmung der side-on-Intensitätsverteilung stets auf das gleiche Meßproblem hinausläuft, wurden deshalb in dieser Arbeit Überlegun-

Sonderdruckanforderungen an I. SANDER, Institut für Angew. Physik, D-2000 Hamburg 36, Jungiusstraße 11. gen angestellt, die zur Entwicklung einer Apparatur führten, welche eine vollautomatische Messung ermöglichte, wobei alle Parameter vor der Messung eingestellt wurden.

Die Messung von Temperatur und Elektronendichte in Funkenkanälen ist nur dann sinnvoll, wenn diese Größen radial aufgelöst gemessen werden, denn nur dann ist es möglich, Aussagen über die Maximaltemperatur zu machen, die in der Achse des Funkenkanals erreicht wird. Das gleiche gilt für die Elektronendichte. Eine der Zielsetzungen der Messungen mit dieser Apparatur war es deshalb, sowohl die axialen als auch die radialen Strukturen der Temperatur und Elektronendichte in der hydrodynamischen Entwicklungsphase von thermischen Funkenkanälen zu studieren.

\section{Meßprinzip}

Zur Messung der zeitlichen Entwicklung von Temperatur- und Elektronendichteverteilung in stromstarken Funken mit Hilfe spektroskopischer Methoden werden in einem Entladungssystem periodisch Funken ausgelöst. Um örtlich aufgelöste Spektroskopie betreiben zu können, wird durch geeignete optische Anordnungen nur das Licht eines kleinen Gebiets des Funkenkanals ausgeblendet und spektral zerlegt. Zwei Photomultiplier registrieren auf ge-

1 V. F. Egorova, V. I. Iasenko, A. A. Mak u. A. I. SadyKovA, Soviet Phys. - Tech. Phys. 7, 242 [1962].

2 H. Tholl, Z. Naturforsch. 22 a, 1068 [1967]. 
trennt einstellbaren Wellenlängenbereichen den zeitlichen Verlauf der Lichtemission. Die Photomultiplierströme werden zu vorwählbaren Zeitpunkten nach Auslösung des Funkens kurzzeitig mit einer Sampling-Anordnung gemessen, wobei die entsprechenden Momentanwerte bis zur Erfassung durch $x y$-Schreiber gespeichert werden können. Werden nun bei aufeinanderfolgenden Funken verschiedene Gebiete ausgeblendet, und wird die Lage des aufgeblendeten Gebietes relativ zur Funkenachse als $x$ Koordinate eines Meßpunktes, die zu einem Zeitpunkt gemessene Lichtintensität als $y$-Koordinate auf einen $x y$-Punktschreiber gegeben, so erhält man unter der Voraussetzung, daß wegen der gleichen Anfangsbedingung die einzelnen Funken weitgehend gleiche Eigenschaften besitzen, axiale und radiale Intensitätsprofile, aus denen nach geeignetem Verfahren ${ }^{1}$ Temperaturen und Elektronendichten bestimmt werden können. Im folgenden sollen die Einzelheiten dieser Apparatur beschrieben werden.

\section{Entladungssystem und elektrischer Stoßkreis}

Die Untersuchung der Funkenkanäle wurde mit Hilfe einer Stoßspannungsapparatur (Abb. 1) im homogenen Feld durchgeführt. Das Entladungssystem $F$ befindet sich in einem evakuierbaren Rezipienten und besteht aus zwei ebenen Elektroden mit $120^{\circ}$ Rogowski-Profil. Als größter Abstand können $3 \mathrm{~cm}$ eingestellt werden. Während die Anode $\mathrm{A}$ über einen Meßwiderstand $R_{\mathrm{M}}$ $(0,1 \mathrm{Ohm})$ mit Masse verbunden ist, wird der Kathode $\mathrm{K}$ eine zum Durchschlag nicht ausreichende negative

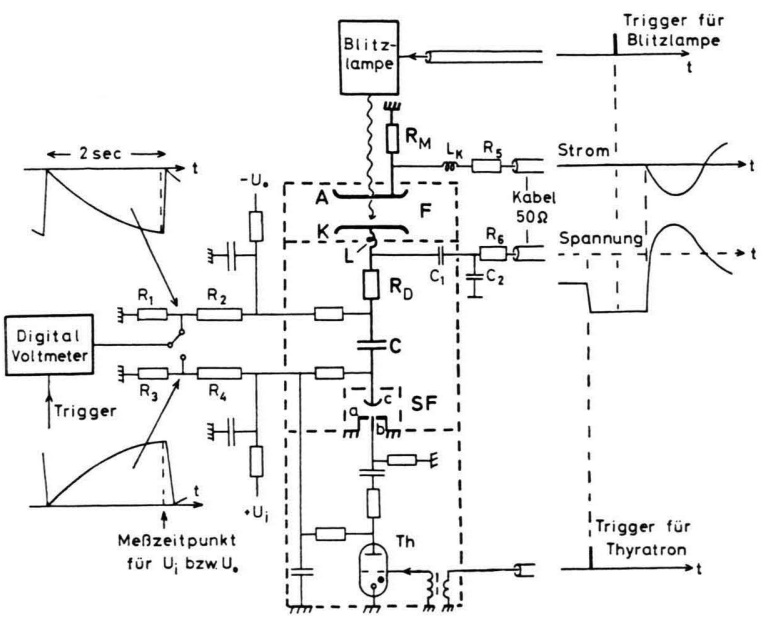

Abb. 1. Schaltbild des elektrischen Stoßkreises (gestrichelt Abschirmung) mit den Meßeinrichtungen für die Impulsformen von Strom und Spannung (rechts) sowie für Ladespannung des Stoßkondensators C (links); weitere Erklärungen im Text.
Spannung $-U_{0}$ zugeführt. Der Stoßkondensator $\mathrm{C}$ wird auf insgesamt $U_{0}+U_{\mathrm{i}}$ aufgeladen. Auf einen Triggerimpuls aus der Impulszentrale zündet das Thyratron Th, das über die Triggerfunkenstrecke (ab) die Schaltfunkenstrecke SF durchschaltet, und damit $\mathrm{C}$ einseitig auf Masse legt. Nach erfolgter Umladung liegt dann unter Vernachlässigung der Brennspannung der Schaltfunkenstrecke die Spannung $U=U_{0}+U_{\mathrm{i}} \cdot C /\left(C+C_{\mathrm{E}}\right)$ an der Entladungsstrecke, wobei $C_{\mathrm{E}}$ die Kapazität der Entladungsstrecke ist. Etwa 200 nsec später, wenn die störenden Einschaltschwingungen abgeklungen sind, wird die Blitzlampe getriggert, deren UV-Blitz durch ein ca. 0,5 $\mathrm{mm}$ großes Loch in der Anode auf die Mitte der Kathode fällt und dort durch Photoeffekt ca. $10^{3}$ Elektronen auslöst. Die Stromstärke des sich daraufhin entwickelnden Funkens kann mit Hilfe des Meßwiderstandes $R_{\mathrm{M}}$ gemessen werden. Wegen des sehr steilen Stromanstiegs muß die induktive Komponente des Spannungsabfalls an $\mathrm{R}_{\mathrm{M}}$ im Meßzweig durch eine Zusatzinduktivität $\mathrm{L}_{\mathrm{k}}$ kompensiert werden. Der Spannungsverlauf an der Kathode wird über den kapazitiven Teiler $C_{1} / C_{2}$ gemessen. Bei der Messung ist jedoch der Spannungsabfall an der Zuleitungsinduktivität $\mathrm{L}$ zu berücksichtigen.

Die Zuführung der Spannungen $U_{0}$ und $U_{\mathrm{i}}$ erfolgt über Siebketten, um trotz der periodischen Entladung von $C$ die Netzgeräte möglichst gleichmäßig zu belasten. Wegen der großen Zeitkonstanten der Siebketten wird $C$ innerhalb einer Periode nicht auf den statischen Endwert aufgeladen. Um trotzdem die im Augenblick der Zündung für den Funken zur Verfügung stehende Spannung messen zu können, werden die Spannungen $U_{0}$ bzw. $U_{\mathrm{i}}$ über Ohmsche Spannungsteiler $\mathrm{R}_{1} / \mathrm{R}_{2}$ bzw. $R_{3} / R_{4}$ jeweils kurz vor der Auslösung des Funkens auf einen Triggerimpuls hin von einem Digitalvoltmeter innerhalb von 20 msec gemessen.

\section{Optischer Aufbau}

In Abb. 2 wurde der prinzipielle Aufbau und ein Blockbild der einzelnen Baugruppen der Apparatur wiedergegeben. Dabei stellt Abb. 2 a den optischen Aufbau in Richtung der Funkenachse und Abb. 2 b senkrecht zur Funkenachse gesehen dar. Der im Entladungssystem ausgelöste Funke $F$ wird über die Linse $L_{1}$ auf den Eintrittsspalt E des Spektrographens M (Bausch \& Lomb) im Verhältnis 1:1 abgebildet. Der Eintrittsspalt ist $1 \mathrm{~mm}$ hoch und weniger als $0,1 \mathrm{~mm}$ breit und läßt daher nur das Licht eines begrenzten Gebietes des $2 \mathrm{~cm}$ langen und im Endstadium der Entwicklung mehrere mm dicken Funkens in den Spektrographen eintreten und sorgt so für die örtliche Auflösung. Das vom Gitter spektral zerlegte Bild des Eintrittsspaltes wird über die Linse $\mathrm{L}_{5}$ in einen Strahlspalter SP abgebildet, wo das Spektrum in zwei Teile getrennt wird, aus denen schließlich zwei Austrittsspalte Spektralbereiche ausblenden, deren Licht von den Photomultipliern PM 1, PM 2 (Valvo 56 TVP) registriert wird. Somit ist eine Ausmessung des abgestrahlten Lichtes auf zwei Wellenlängenbereichen gleichzeitig möglich. 


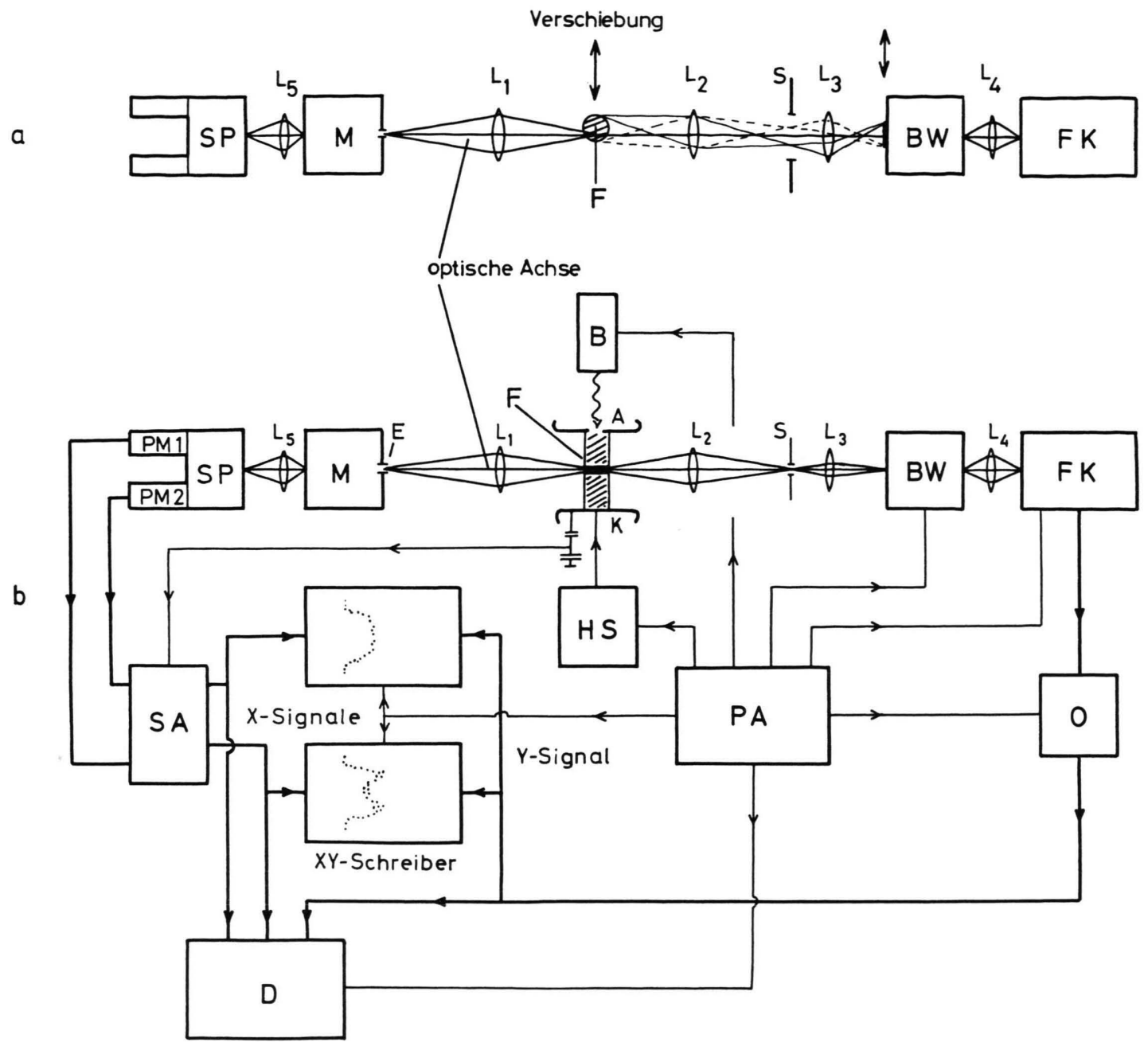

Abb. 2. a) Optischer Aufbau der Apparatur in Richtung der Funkenachse gesehen. b) Optischer Aufbau senkrecht zur Funkenachse gesehen und Blockschaltbild der Apparatur; Signalleitungen wurden dick, Triggerleitungen dünn gezeichnet; die Bedeutung der einzelnen Baugruppen ist im Text erläutert, wobei in allen Kapiteln auf diese Abb. 2 Bezug genommen wird.

Da der Verlauf der Lichtintensität senkrecht zur Funkenachse als Funktion des Abstandes von der Funken. achse gemessen werden soll, muß neben der Intensität auch die Lage des ausgeblendeten Gebiets relativ zur Funkenachse als zweite Meßgröße ermittelt werden. Wegen der Statistik in der Auslösung der Startelektronen sowie wegen statistischer Schwankungen im weiteren Aufbau des Funkens springen die aufeinanderfolgenden Funken in radialer Richtung um etwa einen Funkenradius hin und her, so daß für jeden Funken eine individuelle Ausmessung der Funkenmitte erfolgen muß. Dazu wird der Funkenkanal über eine weitere Linse $\mathrm{L}_{2}$ auf den Spalt $\mathrm{S}$ abgebildet, der einen $1 \mathrm{~mm}$ hohen senkrecht zur Funkenachse liegenden Streifen ausblendet. Dieser Streifen wird über $\mathrm{L}_{3}$ auf die Photokathode der elektronischen Wischkamera BW (RCA 4449 A) abgebildet. Bei einer Wischrichtung parallel zur Funkenachse entsteht wegen der hydrodynamischen
Expansion des Funkenkanals ein keilförmig sich verbreiterndes Wischbild, dessen Spitze die Kanalmitte angibt (Abb. 3).

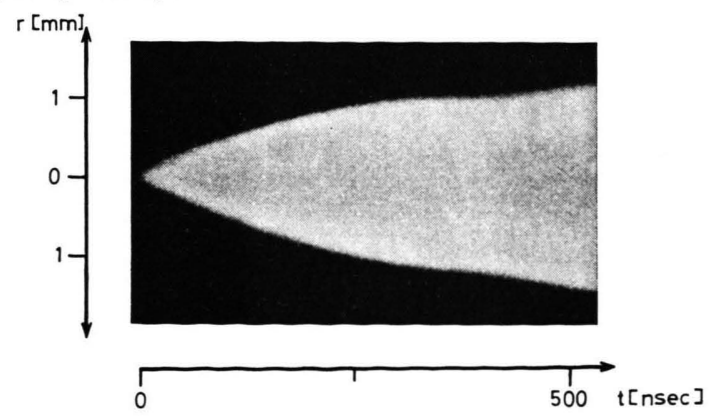

Abb. 3. Mit der elektronischen Wischkamera erhaltenes Wischbild des expandierenden Funkenkanals $\left(\mathrm{H}_{2} ; 460\right.$ Torr, $\mathrm{U}=21,5 \mathrm{kV} ; \mathrm{C}=10 \mathrm{nF}$ ). 


\section{Elektronische Auswertung des Wischbildes}

Um einen voll automatischen Meßbetrieb zu ermöglichen, wird das Wischbild von einer Fernsehkamera FK (System Televisor der Fernseh-GmbH) aufgenommen, aus deren Videosignal elektronisch die Lage der Funkenmitte bestimmt werden kann. Als Kameraröhre dient ein Image Orthicon, das eine ausgezeichnete Lichtempfindlichkeit besitzt, und außerdem bei entsprechender Ansteuerung Bilder etwa $100 \mathrm{msec}$ lang ohne wesentliche Schärfeverluste speichern kann ${ }^{3}$. Entsprechend der Belichtung werden aus der Photokathode Elektronen ausgelöst, die durch ein elektrisches Feld beschleunigt und durch ein axiales Magnetfeld auf eine Speicherfolie fokussiert werden, wo sie nach Auslösung von Sekundärelektronen ein positives Ladungsbild hinterlassen. Das Ladungsbild wird von einem Elektronenstrahl entsprechend der Fernsehnorm rasterförmig abgetastet; dabei werden die positiven Stellen von Elek-

$\mathbf{a}$

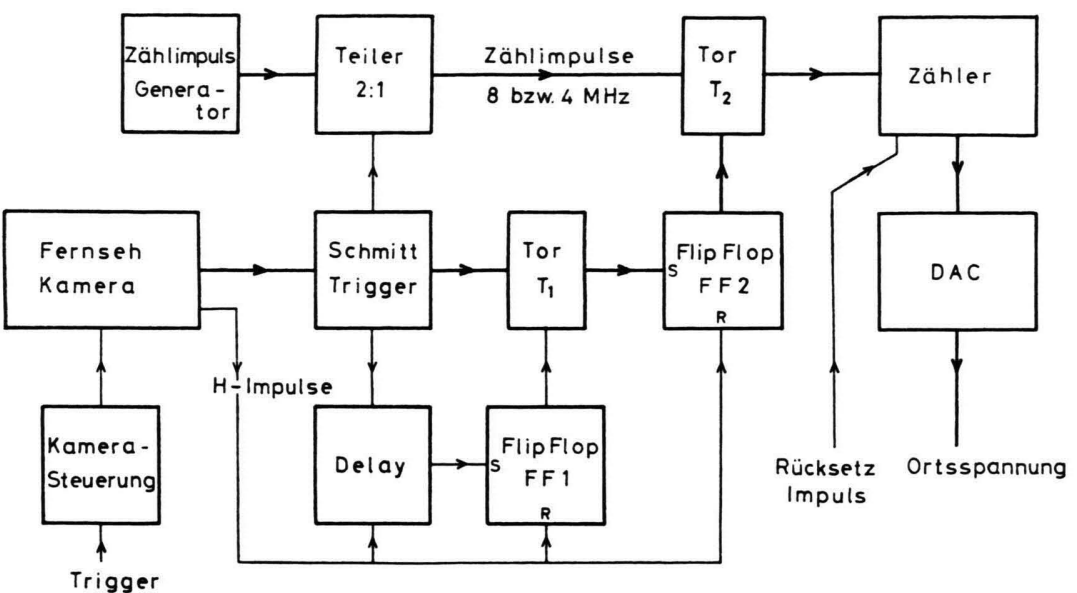

Fernsehbild

und dessen

Abtastung
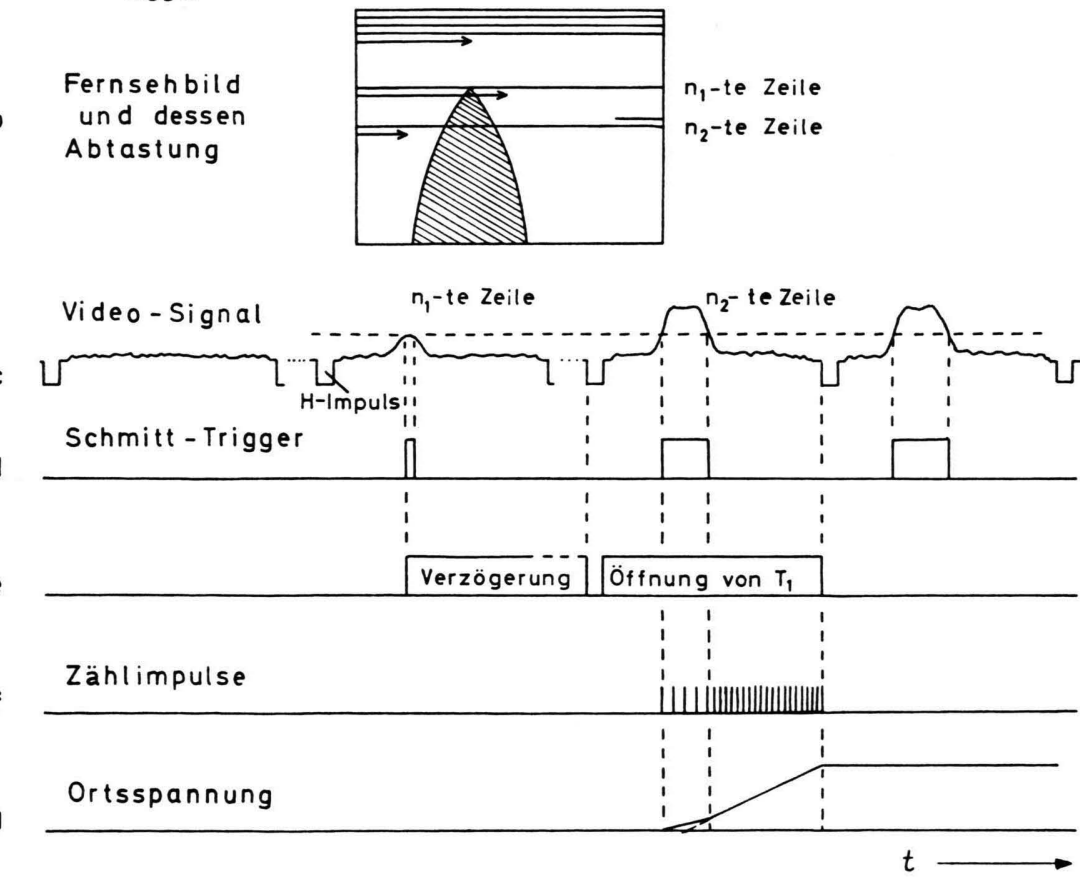

Abb. 4. Blockschaltbild des OrtsSpannungskonverters (in Abb. 2 b Baugruppe 0), mit dessen Hilfe aus dem Fernsehbild die Lage der Funkenachse relativ zur optischen Achse ermittelt und in eine dazu proportionale Spannung umgesetzt wird; genaue Erläuterungen im Text.

3 W. Hopmann, Die Entwicklung einer Kurzzeitkamera hoher Auflösung mit dem Image-Orthikon, Berichte der Kernforschungsanlage Jülich, Nr. 290. 
tronen des Abtaststrahles neutralisiert und die restlichen Elektronen kehren zu einem Auffänger zurück und erzeugen einen Signalstrom, der ein $\mathrm{Maß}$ für die Belichtung der gerade abgetasteten Stelle ist.

Die elektronische Auswertung des von der Fernsehkamera gelieferten Videosignals erfolgt in der in Abb. 2 b mit 0 bezeichneten Baugruppe in der folgenden Weise:

Durch Unterdrückung des abtastenden Elektronenstrahls kann das Ladungsbild gespeichert werden. Von dieser Möglichkeit wird bei der hier beschriebenen Apparatur Gebrauch gemacht. Vor der Auslösung des Funkens wird deshalb die Kamera auf Speicherbetrieb geschaltet. Das einmalig aufleuchtende Wischbild wird gespeichert, und nach Abklingen der elektrischen Störung des Funkens wird mit der Abtastung begonnen. Bei der Abtastung des keilförmigen Wischbildes (Abb. 4 b) ergibt sich ein Videosignal nach Abb. 4c. (Die H-Impulse geben zur Synchronisierung von Bildwiedergabegeräten das Ende der einzelnen Zeilen an.) Während der ersten Zeilen fließt nur Dunkelstrom, bis schließlich in der $n_{1}$-ten Zeile die Spitze des Keiles abgetastet wird, so daß im Videosignal ein Spannungsimpuls erscheint. Bei einer seitlichen Verschiebung (s. Abb. 2 a) der Funkenmitte und damit auch einer Verschiebung der Spitze des Keils im Wischbild ändert sich der Zeitpunkt der Abtastung dieser Spitze innerhalb einer Zeile, so daß aus dem Zeitintervall zwischen Spannungsimpuls und dem nächsten H-Impuls ein $\mathrm{Maß}$ für die Lage der Funkenmitte gewonnen werden kann. Beim Überschreiten eines Schwellwertes spricht der Schmitt-Trigger in Abb. 4 a, $d$ an, der seinerseits eine Verzögerungsstufe (Delay) ansteuert. Die Verzögerungszeit endet mit dem Beginn der $n_{2}$-ten Zeile (Abb. 4 e). Bis zu dieser Zeile ist die Spannungsspitze im Videosignal wegen der ansteigenden Helligkeit im Wischbild erheblich angewachsen, so daß der Schmitt-Trigger hier schon von den sehr steilen Flanken des Spannungsimpulses gesteuert wird, was einen geringeren zeitlichen Jitter des Schmitt-Trigger-Einsatzes zur Folge hat. Nach Ablauf der Verzögerungszeit wird das Tor $\mathrm{T}_{1}$ geöffnet (Abb. $4 \mathrm{e}$ ), so $\mathrm{da} ß$ der nächste eintreffende Schmitt-Trigger-Impuls das Flip-Flop FF2 umschaltet, welches seinerseits das Tor $\mathrm{T}_{2}$ öffnet. Daraufhin gelangen Zählimpulse aus dem Zählimpulsgenerator in einen Digitalzähler bis durch den nächsten H-Impuls das Flip-Flop FF2 wieder zurückgesetzt wird und das Tor $\mathrm{T}_{2}$ weiteren Zählimpulsen den Weg versperrt (Abb. 4 f). Wegen der großen Totzeit der Verzögerungsstufe bleiben die restlichen Zeilen bei der Auswertung unberücksichtigt. Während der Zeit, in der das Videosignal die Ansprechschwelle des Schmitt-Triggers überschreitet, wird die Zählimpulsfrequenz im Teiler 2: 1 halbiert - bei symmetrischem Impulsverlauf ist das gleichbedeutend mit einem Zählbeginn in der Mitte des Impulses -, so daß der Zählerstand dann proportional zum Abstand von der Mitte des Spannungsimpulses bis zum nächsten H-Impuls ist, und damit schließlich die Lage der Funkenmitte angibt.

In einem Digital-Analog-Wandler DAC erfolgt die Umsetzung des Zählerstands in einen analogen Spannungswert (Abb. $4 \mathrm{~g}$ ), der den $x y$-Schreibern als "Ortsspannung“ zugeführt wird. Der Knick im linearen Anstieg der Ortsspannung rührt von der halben Impulsfrequenz während der Schmitt-Trigger-Schaltzeit her. Nach der Abtastung des Wischbildes wird die Fernsehkamera von der Kamerasteuerung wieder in den Speicherzustand geschaltet.

\section{Elektronische Auswertung der Multiplier- ströme}

Um den zeitlichen Ablauf der einzelnen Funkenstadien zu verfolgen, sollen die Intensitätsprofile senkrecht zur Funkenachse zu verschiedenen Zeiten der Funkenentwicklung aufgenommen werden. Es wurde hier ein Verfahren angewendet, bei dem nacheinander die vollständigen Profile zu vorgegebenen Zeitpunkten ausgemessen werden. Dazu ist es nötig; 1. die Lage des ausgeblendeten Gebietes über den Funkendurchmesser zu variieren und seine jeweilige Lage bezüglich der Funkenachse für jeden Funken erneut zu vermessen,

2. jeweils die Lichtintensität zu einem festen Zeitpunkt nach Auslösung des Funkens zu messen und den Meßwert bis zur Registrierung durch einen $x y$-Schreiber zu speichern.

Die Lösung der ersten Aufgabe wurde im vorangegangenen Abschnitt bereits beschrieben. Zur Lösung der zweiten Aufgabe werden einer SamplingAnordnung (s. Abb. $5 \mathrm{a}$ und in Abb. $2 \mathrm{~b}$ die Baugruppe SA) die dem zeitlichen Verlauf der Lichtintensität proportionalen Multipliersignale (Abb. $5 \mathrm{~d}$ ) zugeführt. Dort ist die im Signalweg liegende Torschaltung - eine Diodenbrücke - normalerweise im gesperrten Zustand. Nur für die kurze Zeit $(0,3$ nsec) eines Öffnungsimpulses werden alle Dioden in den Durchlaßzustand geschaltet, so daß dann der 


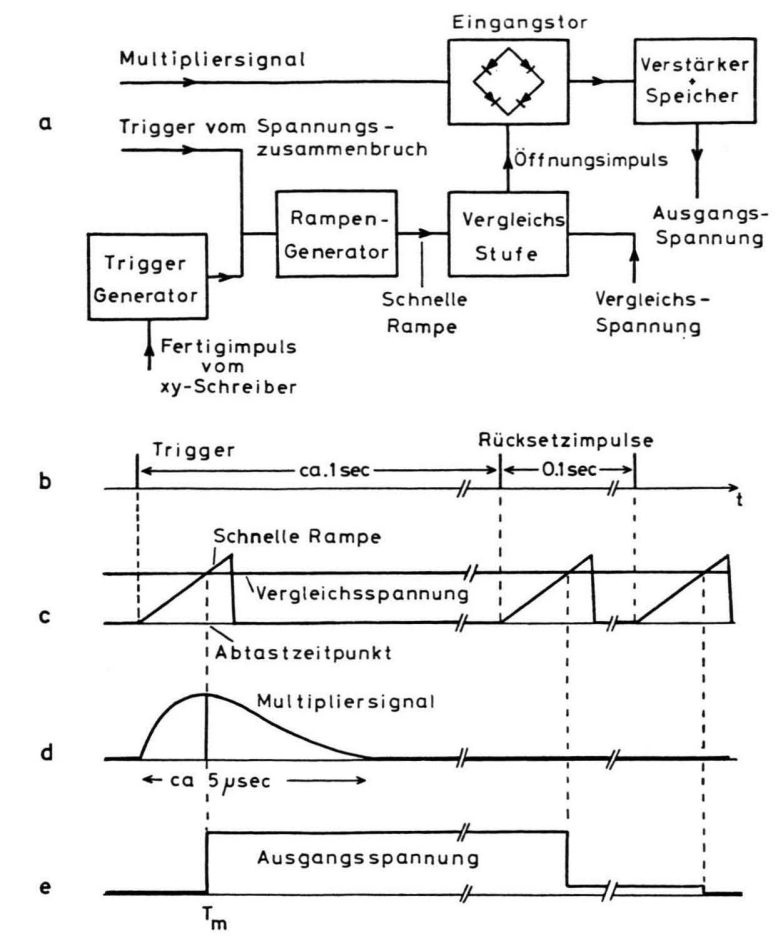

Abb. 5. Blockschaltbild und Impulsverläufe der Sampling-Anordnung (Baugruppe SA in Abb. 2 b) ; weitere Erklärungen im Text.

Signalweg geschlossen ist und ein Kondensator auf eine Spannung aufgeladen werden kann, die proportional zur Amplitude des Photomultiplier-Signals zum Meßzeitpunkt ist. Anschließend wird die Verbindung wieder unterbrochen, und die Spannung am Kondensator steht nach Verstärkung durch einen Halteverstärker (Speicher) am Ausgang der Sampling-Anordnung für die Erfassung durch den $x y$ Schreiber zur Verfügung.

Zur Gewinnung des Öffnungsimpulses für das Eingangstor wird über einen kapazitiven Teiler (in Abb. $1 C_{1} / C_{2}$ ) vom Spannungszusammenbruch, und damit vom Beginn des stromstarken Funkens, eine schnell ansteigende Spannung, die Rampenspannung, gestartet (Abb. 5 c), die in einer Vergleichsstufe mit einer extern zugeführten Gleichspannung verglichen wird. Im Augenblick der Übereinstimmung wird der 0,3 nsec lange Öffnungsimpuls für die Diodenbrücke erzeugt. Dabei kann die Verzögerung des Zeitpunktes der Abtastung entweder durch Variation der Anstiegsgeschwindigkeit der Rampenspannung oder durch unterschiedliche Höhe der Vergleichsspannung in einem weiten Bereich von nsec bis zu msec verändert werden. Nach der Registrierung der Aus- gangsspannung durch den $x y$-Schreiber erhält die Sampling-Anordnung aus einem Trigger-Generator Rücksetzimpulse (Abb. $55 \mathrm{~b}$ ), die dann die Ausgangsspannung auf 0 zurücksetzen (Abb. $5 \mathrm{e}$ ), da zu diesem Zeitpunkt kein Multiplierstrom mehr fließt, so daß das Multipliersignal am Eingangstor $\mathrm{O}$ ist. Prinzipiell würde für die Rücksetzung der Ausgangsspannung der Sampling-Anordnung ein einziger Trigger-Impuls genügen. Bedingt durch Unsymmetrien des Eingangstors ist es jedoch in der Regel notwendig, mehrere Impulse zu benutzen, so daß eine stufenweise Rücksetzung erfolgt. Als Sampling-Anordnung (in Abb. 2 b Block SA) wurde der zum Siemens-Oszillographen M 214 gehörende ZweiKanal-Sampling-Einschub M 719-A 1 benutzt, wobei auf die zwei Sampling-Kanäle die Signale der beiden Photomultiplier geschaltet wurden.

\section{Automatische Steuerung der Apparatur}

Wie schon erwähnt, muß zur Aufnahme der Intensitätsprofile die Lage des ausgeblendeten Gebiets relativ zur Funkenachse über den gesamten Funkendurchmesser verschoben werden. Da das statistische Hin-undHerspringen des Funkens dazu nicht ausreicht, wird in der hier beschriebenen Apparatur der ganze Rezipient und damit auch das Entladungssystem mit dem Funken relativ zur im Raum festliegenden optischen Achse durch einen Motorantrieb verschoben (s. auch Abb. 2). Eine Automatik sorgt dabei dafür, daß der Funke im Beobachtungsbereich bleibt. Wird nämlich der Funkenkanal aus der optischen Achse herausgeschoben, dann registrieren die Photomultiplier keine Lichtintensität mehr und das Ausgangssignal der Sampling-Anordnung bleibt bei der Abtastung 0. Ist dies mehrmals hintereinander der Fall, dann wird die Antriebsrichtung für die Verschiebung des Rezipienten umgekehrt, so daß der Funke wieder in die optische Achse hineingeschoben wird.

Ein Digitalzähler registriert die Zahl der Funken, die zur Aufnahme einer radialen Verteilung gezündet wurden. In der Regel wurden 256 oder 512 oder 1024 Funken zur Darstellung einer Verteilungskurve gezündet. Nach Erreichen einer dieser Zahlen wird die Messung der betreffenden Verteilung abgebrochen und durch Veränderung des Rampenanstiegs oder der Vergleichsspannung der Sampling-Anordnung SA (Abb. 5) ein anderer Meßzeitpunkt $T_{\mathrm{m}}$ eingestellt. Ferner erhalten die Papiervorschübe der $x y$-Schreiber einen Schaltimpuls, so daß das Registrierpapier um ca. $10 \mathrm{~cm}$ verschoben wird. Danach beginnt die Messung der nächsten Radialverteilung. Da die Messung periodisch mit zwei Sekunden erfolgte, wurden zur Bestimmung einer Verteilung ca. 10-40 Minuten je nach Funkenzahl benötigt.

$\mathrm{Zu}$ welchen Meßzeitpunkten $T_{\mathrm{m}}$ das vom Funken abgestrahlte Licht untersucht werden soll, kann vor Be- 
ginn der gesamten Messung an einem Programmpult für 30 Zeitpunkte vorprogrammiert werden, so daß die eigentliche Messung dann vollautomatisch abläuft.

\section{Zusammenwirken der einzelnen Baugruppen (Impulsfahrplan)}

Um einen Überblick über das Zusammenwirken der verschiedenen Baugruppen und über den Meßablauf innerhalb einer Periode zwischen zwei Punkten zu erhalten, wurde in Abb. $2 \mathrm{~b}$ das Blockschaltbild und in Abb. 6 der Impulsplan der Anlage dargestellt. Mit einem zeitlichen Abstand von in der

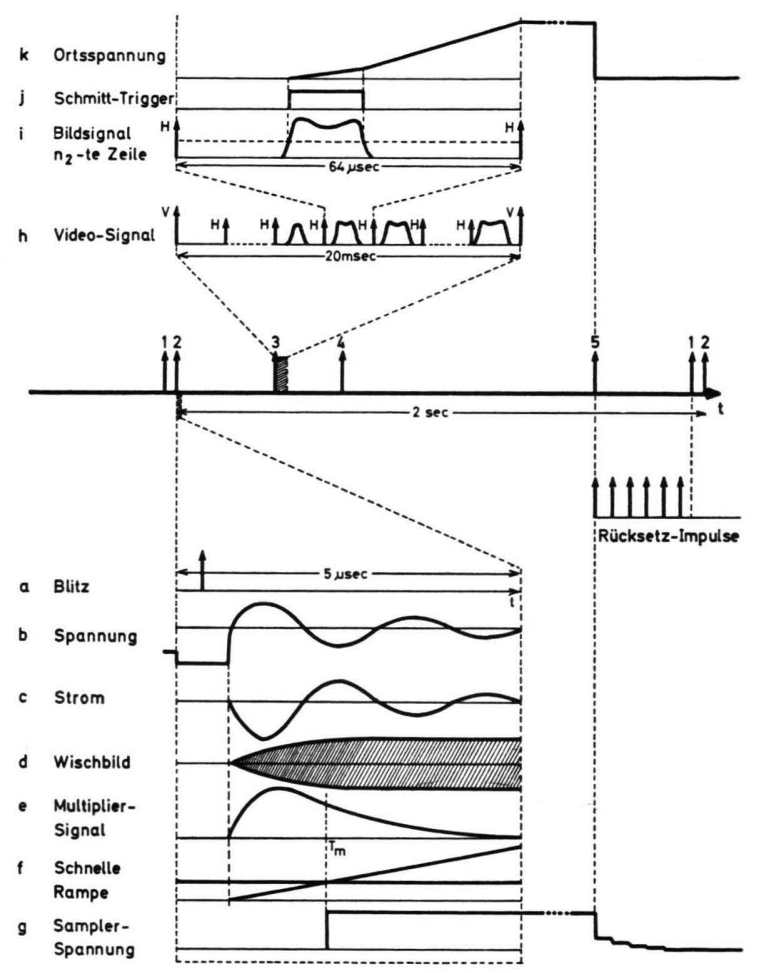

Abb. 6. Impulsfahrplan der Apparatur, der den zeitlichen Ablauf der einzelnen Messungen innerhalb einer Periode zwischen zwei aufeinanderfolgenden Funken angibt.

Regel zwei sec werden durch die Impulsanlage PA (Abb. $2 \mathrm{~b}$ ) periodisch durch Triggerung des Hochspannungsstoßkreises HS und durch Zünden des UV. Lichtblitzes B (s. auch Abb. 1) die Funkenkanäle in der Entladungsstrecke AK gezündet. In Abb. 6 sind dies die Zeitpunkte 2 auf der in der Mitte liegenden Hauptzeitachse. Etwa 30 msec vor der Zündung des Funkens wird das Digital-Voltmeter getriggert, welches die Ladespannung des Stoßkondensators wenige Millisekunden vor der Funkenzündung mißt (Zeit- punkt 1). In dem an Zeitpunkt 2 sich anschließenden schraffierten Zeitintervall von ca. $5 \mu$ sec, welches gedehnt herausgezeichnet wurde, läuft die Funkenentwicklung ab. Etwa 200 nsec nach dem Hochspannungsstoß (Abb. 6 b) wird der Blitz (Abb. 6 a) gezündet. Der Entladungsmechanismus benötigt bei den hier verwendeten Überspannungen von 20 bis $30 \%$ etwa 300 nsec bis zum Zünden des stromstarken Funkens, welches sich durch den Zusammenbruch der Spannung (Abb.6b) und den Anstieg des Stromes (Abb. 6 c) markiert.

Die Zeitablenkung der elektronischen Wischkamera BW wird gleichzeitig mit dem UV-Blitz gestartet, so daß das in Abb. 6 d gezeigte Wischbild des expandierenden Kanals synchron mit dem Stromimpuls entsteht. Zur selben Zeit registrieren auch die Photomultiplier den emittierten Lichtimpuls des Funkens (Abb.6 e). Der Spannungsimpuls startet die schnelle Rampe des Sampling-Einschubes SA (Abb. $6 \mathrm{f}$ ), so daß zum Zeitpunkt $T_{\mathrm{m}}$ die Amplitude des Multipliersignals gemessen wird und die Ausgangsspannung der Sampling-Anordnung auf den entsprechenden Wert ansteigt (Abb. $6 \mathrm{~g}$ ).

Die Abtastung des auf dem Target der Fernsehkamera FK gespeicherten Wischbildes beginnt zum Zeitpunkt 3 etwa 100 msec nach 2. Die Zeitdauer der Abtastung eines Fernsehbildes beträgt $20 \mathrm{msec}$ und wurde in Abb. $6 \mathrm{~h}$ gedehnt dargestellt. Zwischen den H-Impulsen tritt in der $n_{2}$-ten Zeile (s. a. Abb. 4) im Videosignal derjenige Impuls auf (Abb. 6i), welcher den Schmitt-Trigger schaltet (Abb. $6 \mathrm{j}$ ). Schließlich zeigt Abb. $6 \mathrm{k}$ noch die Entstehung der Ortsspannung, deren Endwert am Ende der $n_{2}$-ten Zeile erreicht ist.

Nach Beendigung der Abtastung des Wischbildes werden zum Zeitpunkt 4 die $x y$-Schreiber von der Pulsanlage getriggert. Die Schreiber benötigen etwa eine halbe Sekunde, um sich auf die zwei Spannungswerte (Ortsspannung, und Ausgangsspannung der Sampling-Einheit) einzustellen. Zum Zeitpunkt 5 ist der Abgleich beider Schreiber erfolgt und jeder hat an der betreffenden Stelle $x, y$ einen Punkt gedruckt. Jetzt können durch die Rücksetzimpulse die zwei Spannungswerte (Abb.6k und $6 \mathrm{~g}$ ) gelöscht werden. Der die Rücksetzimpulse erzeugende Triggergenerator (Abb. 5 a) wird zum Zeitpunkt 1, bei dem die neue Periode beginnt, ausgeschaltet.

Die in Abb. $2 \mathrm{~b}$ mit $\mathrm{D}$ bezeichnete Baugruppe stellt einen Analog-Digital-Wandler mit nachfolgender Locheransteuerung und Lochstreifenstanzer dar. 
Dadurch ist es möglich, die drei Spannungswerte - Ortsspannung und Ausgangsspannungen der beiden Sampling-Kanäle - sofort digital zu erfassen.

\section{Gemessene Verteilungskurven}

Abb. 7 zeigt als Beispiel für die Darstellung der Ergebnisse die von einem $x y$-Schreiber gedruckten Intensitätsverteilungen senkrecht zur Achse eines Wasserstoffunkens (side-on-Verteilungen) zu verschiedenen Zeiten der hydrodynamischen Entwicklung (Expansionsphase) und in der Abkühlungsphase (after glow) des Funkens. Die Strahlung wurde im Brems- und Rekombinationskontinuum bei $\lambda=6000 \AA$ registriert. Die Verteilungen werden
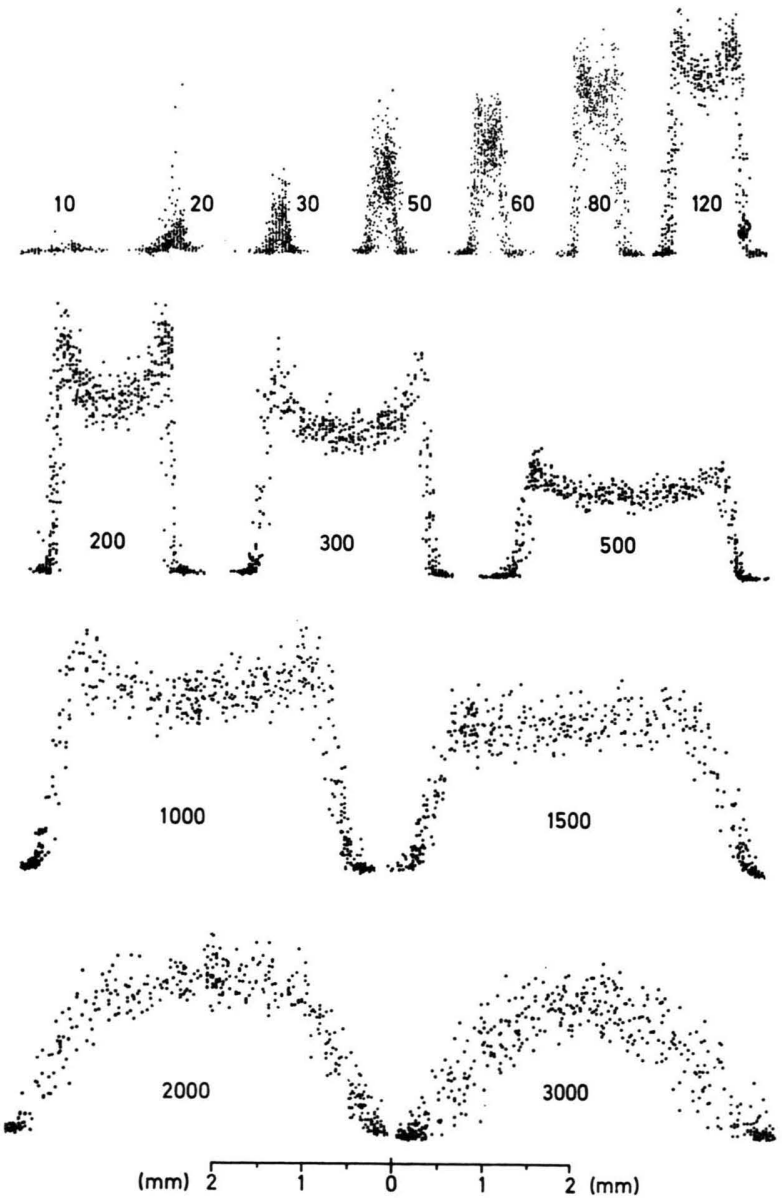

Abb. 7. Zu verschiedenen Zeiten der Funkenentwicklung gemessene Intensitätsverteilungen senkrecht zur Funkenachse (side-on) in Wasserstoff bei $\lambda=6000 \AA$; Parameter ist die Zeit in nsec; experimentelle Daten: Druck: $p=460$ Torr; Stoßkondensator: $C=10 \mathrm{nF}$; Ladespannung des Stoßkondensators: $U=21,5 \mathrm{kV}$. also in Form einer Punktverteilung wiedergegeben, wobei jeder Punkt aus der Messung an einem Funken hervorgegangen ist. In der Streuung der Meßpunkte spiegelt sich die Reproduzierbarkeit der einzelnen Funken wider. Die Streuung der Intensität hängt stark von den experimentellen Bedingungen $\mathrm{ab}$, beträgt jedoch im Mittel etwa $\cong 10 \%$. In Abb. 7 wurde von der Verteilung zum Zeitpunkt 1000 nsec an die Empfindlichkeit um das dreifache vergrößert.

Auf die physikalischen Gründe für die Form dieser Verteilungen wird an dieser Stelle nicht eingegangen, da dies bereits ausführlich in ${ }^{1}$ und ${ }^{2}$ erfolgte. Gut erkennbar ist jedoch die Expansion des Funkenkanals von einem ca. 3/10 mm dünnen Faden (20 nsec) bis zu einem etwa $4 \mathrm{~mm}$ dicken Kanal im Endstadium der Aufweitung (1500 nsec).

Für die weitere Auswertung dieser Verteilungen ist es erforderlich, zunächst eine gemittelte Kurve

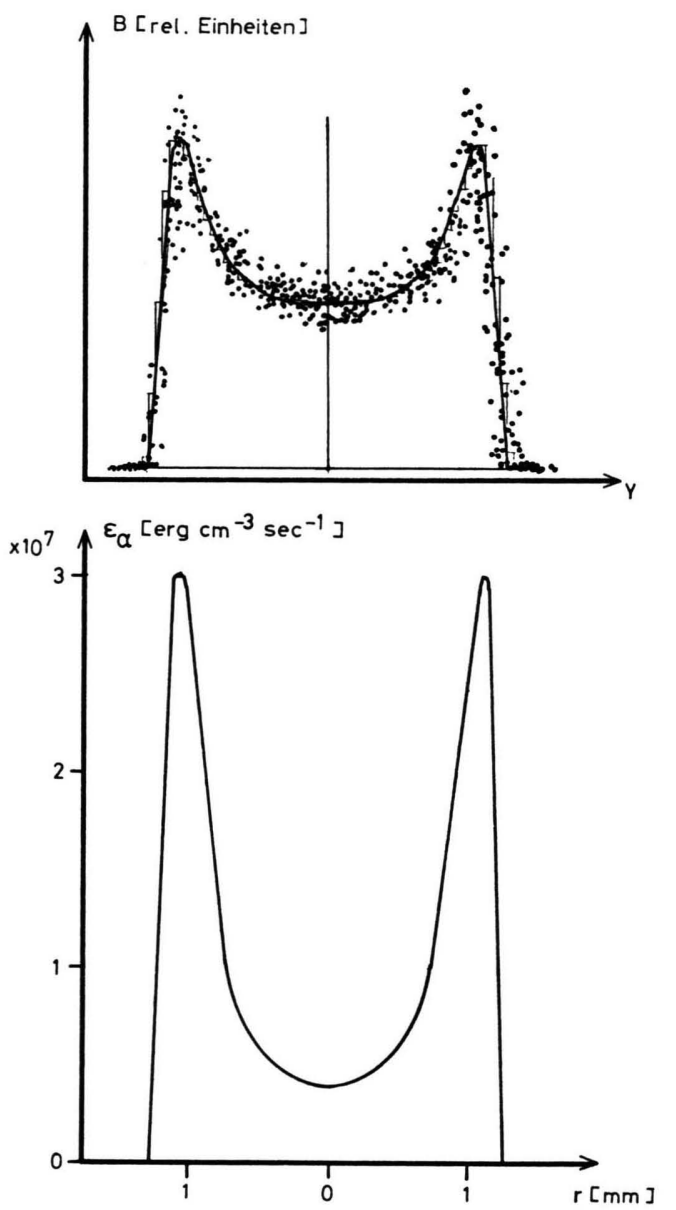

Abb. 8. Gegenüberstellung einer side-on-Verteilung und der dazugehörenden radialen Intensitätsverteilung, die sich nach Lösung der Abelschen Integralgleichung ergibt. 
durch die Punkte hindurchzulegen. Aus dieser gemittelten side-on-Verteilung kann dann durch numerische Lösung der Abelschen Integralgleichung die radiale Verteilung der Intensität berechnet werden. Diese Rechnungen wurden am Elektronenrechner TR 4 der Universität Hamburg nach einem in ${ }^{1}$ beschriebenen Verfahren durchgeführt. Abb. 8 zeigt als Beispiel die Gegenüberstellung einer side-on-Verteilung und der dazugehörenden radialen Intensitätsverteilung nach Lösung der Abelschen Integralgleichung.

Um die mit der Mittelung der Punktverteilungen verbundenen Unsicherheiten zu reduzieren, wurden bei einigen Messungen die Koordinaten der einzelnen Punkte direkt digital in einen Lochstreifen gestanzt (Baugruppe D in Abb. 2 b) und die Mittelung dann mit Hilfe des TR 4-Rechners durchgeführt. Da- bei zeigte es sich jedoch, daß mit einer erheblich größeren Punktdichte gearbeitet werden mußte, um zu brauchbaren "glatten“ gemittelten Kurven zu gelangen, so daß dadurch die Meßzeit stark anstieg.

Die weitere Auswertung der berechneten Radialverteilungen zur Ermittlung der Temperatur- und Elektronendichteverteilungen erfolgte nach dem Normtemperaturverfahren, aus den absoluten Intensitäten von Kontinuums- und Linienstrahlung sowie aus dem Verhältnis von Linien- zu Kontinuumsstrahlung. Diese Verfahren wurden bereits in ${ }^{2}$ genauer beschrieben.

Herrn Prof. Dr. H. RaEther danken wir für die Förderung dieser Arbeit. Der Bundesminister für wissenschaftliche Forschung hat uns in dankenswerter Weise die Mittel für den Aufbau dieser Apparatur zur Verfügung gestellt.

\title{
Thermalisierung und zeitliche Entwicklung der Elektronendichte und Temperatur von Funkenkanälen in Wasserstoff
}

\author{
H. ThOLL \\ Institut für Angewandte Physik der Universität Hamburg \\ (Z. Naturforsch. 25 a, 420-429 [1970] ; eingegangen am 17. Dezember 1969)
}

\begin{abstract}
In a streamer initiated pre-discharge channel in $\mathrm{H}_{2}$ the axial and radial electron density distributions were measured at different times of the channel development. It was found that at any point in the channel where the electron density reaches $\simeq 2 \cdot 10^{17} \mathrm{~cm}^{-3}$ an immediate thermalisation of the plasma at this point takes place in a time of $\cong 10$ nsec. Due to the inhomogeneous radial and axial electron density distribution in a streamer initiated discharge channel the thermalisation begins at first in a constricted region of the channel.

The further development of the electron density and temperature distributions in the thermalized channel was studied by means of spectroscopic plasma diagnostic methods in channels initiated by different overvoltages. These experiments have shown that the inhomogeneous axial electron density distribution is equalized after a time of $\cong 100$ nsec. The maximum electron density of $\cong 1-2 \cdot 10^{18}$ $\mathrm{cm}^{-3}$ is reached in the channel axis immediately after the thermalisation of the channel whereas the temperature increases during the time of the highest energy input to the maximum of $\cong 5-6$ $\cdot 10^{4} \mathrm{~K}$.
\end{abstract}

\section{Einleitung}

In früheren Arbeiten wurde die axiale Verteilung der Intensität des emittierten Lichtes von Funkenkanälen untersucht. Dies erfolgte in älteren Experimenten mit Hilfe der photographischen Beobachtung ${ }^{1-3}$, oder es wurden Drehspiegelanordnungen

Sonderdruckanforderungen an I. SANDER, Institut für Angewandte Physik der Universität Hamburg, D-2000 Ham. burg 36, Jungiusstraße 11.

1 W. Holzer, Z. Phys. 77, 676 [1932].

2 K. R. Allen u. K. Phillips, Proc. Roy. Soc. London 278 , 168,188 [1964]. benutzt, die auch die Durchführung zeitaufgelöster Spektroskopie gestatteten ${ }^{2}$. Eine Fortsetzung dieser Untersuchungen wurde in neueren Arbeiten mit Hilfe der Photomultipliertechnik ${ }^{4}$ und durch den Einsatz elektronischer Bildverstärker und Bildwandler-Wischkameras durchgeführt ${ }^{5-7}$. Alle diese Untersuchungen ergaben, daß insbesondere bei Funken, die mit hö-

${ }^{3}$ H. Tholl, Z. Naturforsch. 19 a, 704 [1964].

${ }^{4}$ H. Tholl, Proc. 7th Int. Conf. Iionization Phenomena in Gases, Belgrad 1, 620 [1965].

5 K. H. Wagner, Z. Phys. 189, 465 [1966].

6 J. Koppitz, Z. Naturforsch. 22 a, 1089 [1967].

7 A. A. Doran, Z. Phys. 208, 427 [1968]. 